\title{
SUPPORTING INFORMATION-DFT CALCULATIONS
}

\author{
Friedel-Crafts Alkylation of Indoles with p-Quinols. The role of hydrogen \\ bonding of water for the desymmetrization of the cyclohexadienone system.
}

\author{
Carolina García-García,${ }^{\dagger}$ Laura Ortiz-Rojano, ${ }^{\dagger}$ Susana Álvarez, ${ }^{*}$ Rosana Álvarez, ** \\ María Ribagorda ${ }^{*, \dagger}$ and M. Carmen Carreño ${ }^{*, \dagger}$ \\ † Departamento de Química Orgánica, Facultad de Ciencias, Universidad Autónoma de Madrid, C/ \\ Francisco Tomás y Valiente no 7, Cantoblanco 28049-Madrid.` Departamento de Química Orgánica, \\ Facultad de Química, Universidade de Vigo, CINBIO, Lagoas-Marcosende s/n, 36310 Vigo, Spain
}

DFT calculations with the hybrid WB97XD (with dispersion correction) ${ }^{1}$ have been carried out using Gaussian $09^{2}$ to locate and characterize the stationary points on the potential energy surface at the $6-31 \mathrm{G}^{*}$ level. The optimized geometries have been characterized by harmonic analysis, and the nature of the stationary points determined according to the number of negative eigenvalues of the Hessian matrix. In several doubtful cases, internal reaction coordinates (IRCs) have been followed from the transition structures to verify the proper connections with reactants and products. ${ }^{3}$ Zero-point vibration energies (ZPVE) and thermal corrections (at $298 \mathrm{~K}, 1 \mathrm{~atm}$ ) to the energy have been estimated using the computed frequencies applying the free particle, harmonic oscillator and rigid rotor approximations at the high temperature limit in a canonical ensemble. Solvation effects (to simulate the experimentally used $\mathrm{CH}_{3} \mathrm{CN}$ ) have been also included as single point corrections to the gas-phase free energy of the optimized structures computed with the $\omega$ B97XD functional with the self-consistent reaction field (SCRF) method using the polarizable continuum model $(\mathrm{PCM})^{4}$ as implemented in Gaussian09.

Comprehensive DFT-based calculations were carried out using as model system the reaction of p-quinol 1a and indole 2a catalyzed by $(R)$-BINOL-3,3'-(9-anthracenyl) phosphoric acid $(R)$-12h leading to the desymmetrization product. To address the stereoselectivity of the reaction, two descriptors will be used, which correspond to the face-selectivity and $\beta$-carbon selectivity, respectively. In all experimental

\footnotetext{
${ }^{1}$ Chai, J.-D.; Head-Gordon, M. Phys. Chem. Chem. Phys. 2008, 10, 6615.

${ }^{2}$ Frisch, M. J.; Trucks, G. W.; Schlegel, H. B.; Scuseria, G. E.; Robb, M. A.; Cheeseman, J. R.; Scalmani, G.; Barone, V.; Mennucci, B.; Petersson, G. A.; Nakatsuji, H.; Caricato, M.; Li, X.; Hratchian, H. P.; Izmaylov, A. F.; Bloino, J.; Zheng, G.; Sonnenberg, J. L.; Hada, M.; Ehara, M.; Toyota, K.; Fukuda, R.; Hasegawa, J.; Ishida, M.; Nakajima, T.; Honda, Y.; Kitao, O.; Nakai, H.; Vreven, T.; Montgomery, J. A.; Peralta, J. E.; Ogliaro, F.; Bearpark, M.; Heyd, J. J.; Brothers, E.; Kudin, K. N.; Staroverov, V. N.; Kobayashi, R.; Normand, J.; Raghavachari, K.; Rendell, A.; Burant, J. C.; Iyengar, S. S.; Tomasi, J.; Cossi, M.; Rega, N.; Millam, J. M.; Klene, M.; Knox, J. E.; Cross, J. B.; Bakken, V.; Adamo, C.; Jaramillo, J.; Gomperts, R.; Stratmann, R. E.; Yazyev, O.; Austin, A. J.; Cammi, R.; Pomelli, C.; Ochterski, J. W.; Martin, R. L.; Morokuma, K.; Zakrzewski, V. G.; Voth, G. A.; Salvador, P.; Dannenberg, J. J.; Dapprich, S.; Daniels, A. D.; Farkas; Foresman, J. B.; Ortiz, J. V.; Cioslowski, J.; Fox, D. J., Gaussian 09, Revision B.01, Wallingford CT, 2009.

${ }^{3}$ (a) Fukui, K. Acc. Chem. Res. 1981, 14, 363. (b) Gonzalez, C.; Schlegel, H. B. J. Phys. Chem. 1990, $94,5523$.

${ }^{4}$ (a) Tomasi, J.; Persico, M. Chem. Rev. 1994, 94, 2027. (b) Cossi, M.; Scalmani, G.; Rega, N.; Barone, V. J. Chem. Phys. 2002, 117, 43. (c) Tomasi, J.; Mennucci, B.; Cammi, R. Chem. Rev. 2005, 105, 2999.
} 
cases, compound $\mathbf{3 a}$ was characterized as a single diastereoisomer resulting from a Si face-pro- $R \mathrm{C} \beta$ or Re face-pro-S C $\beta$ selectivity in the $(1,4)$-addition of indole $\mathbf{2 a}$ to the less hindered face of $p$-quinol $\mathbf{1 a}$ (OH containing face), anti to the $\mathrm{CH}_{3}$ group. In order to carry out conveniently the DFT study, we selected the reaction on the less hindered face as this is the experimentally obtained diastereoface-selective reaction. Therefore, enantiomers of the Friedel-Crafts-type reaction product $\mathbf{3 a}$ would be formed by reaction of indole to the $\mathrm{C} \beta$ of 1 a with opposite topicities (i.e., Si face-pro- $R \mathrm{C} \beta$ or $R e$ face-pro- $S \mathrm{C} \beta$ ). In all cases, the two-point coordination Akiyama's model, which has been described for coordination of the BINOL phosphoric acid catalyst to the two reagents has been adopted. For this particular case, the coordination mode involves a double hydrogen bond established between the acidic position of catalyst $\mathbf{1 2} \mathbf{h}$ and $p$-quinol $\mathbf{1 a}$, and between the basic position of catalyst $\mathbf{1 2} \mathbf{h}$ and $\mathrm{N}-\mathrm{H}$ of indole $\mathbf{2 a}$ (Figure 1). ${ }^{5,6}$

Figure 1. Representation of coordination modes of model reactants $p$-quinol $\mathbf{1 a}$ and indole $\mathbf{2 a}$ to the catalyst $[(R)$ BINOL-3,3'-(9-anthracenyl)-phosphoric acid 12h] and structures of the four possible stereoisomers of the reaction product.

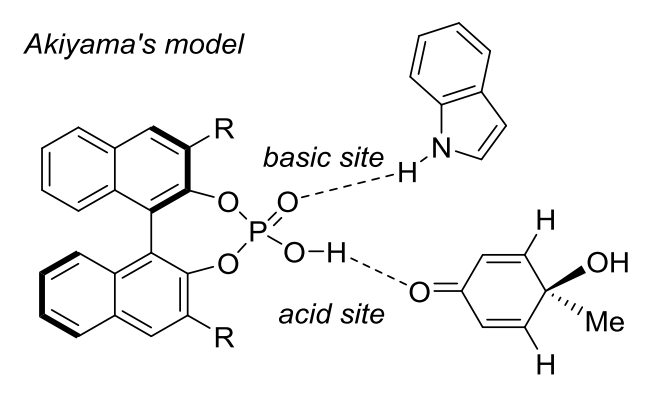

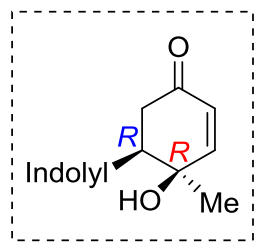

$(4 R, 5 R)$

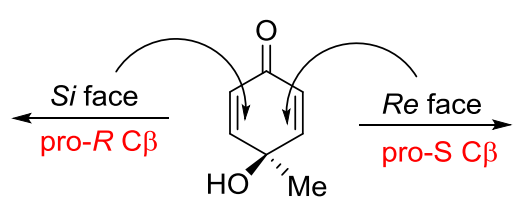

. -

$(4 S, 5 S)$

\section{EFFECT OF THE WATER MOLECULES: WATER AND NON-WATER ASSISTED MODELS}

In order to estimate the effect of the water molecules in the reaction, both the water and the nonwater-assisted variants approaches have been computed of the Re face-pro-S C $\beta$ (Figure 2). In Table 1 are shown the water and the non-water-assisted structures of: entry 1) $p$-quinol associated with $(R)$ 12h (I), entry 2) the transitions state that incorporated the indole (TS $\left.\mathbf{I}_{-I I}\right)$, entry 3 ) the final adduct $3 \mathbf{a}$ associated $(R)-\mathbf{1 2 h}($ II).

\footnotetext{
${ }^{5}$ Akiyama, T.; Itoh, J.; Yokota, K.; Fuchibe, K. Angew. Chem. Int. Ed. 2004, 43, 1566-1568.

${ }^{6}$ Parmar, D.; Sugiono, E.; Raja, S.; Rueping, M. Chem. Rev. 2014, 114, 9047-9153.
} 
Table 1. Representation of the structures corresponding to the water and the non-water assisted phosphoric acid-promoted $[(R)-\mathbf{1 2 h}] \mathrm{FC}$ reaction between $p$-quinol 1a and indole $\mathbf{2 a}$ (wB97XD/6-31G*, in gas phase). Relevant atom distances (in $\AA$ ) are shown.

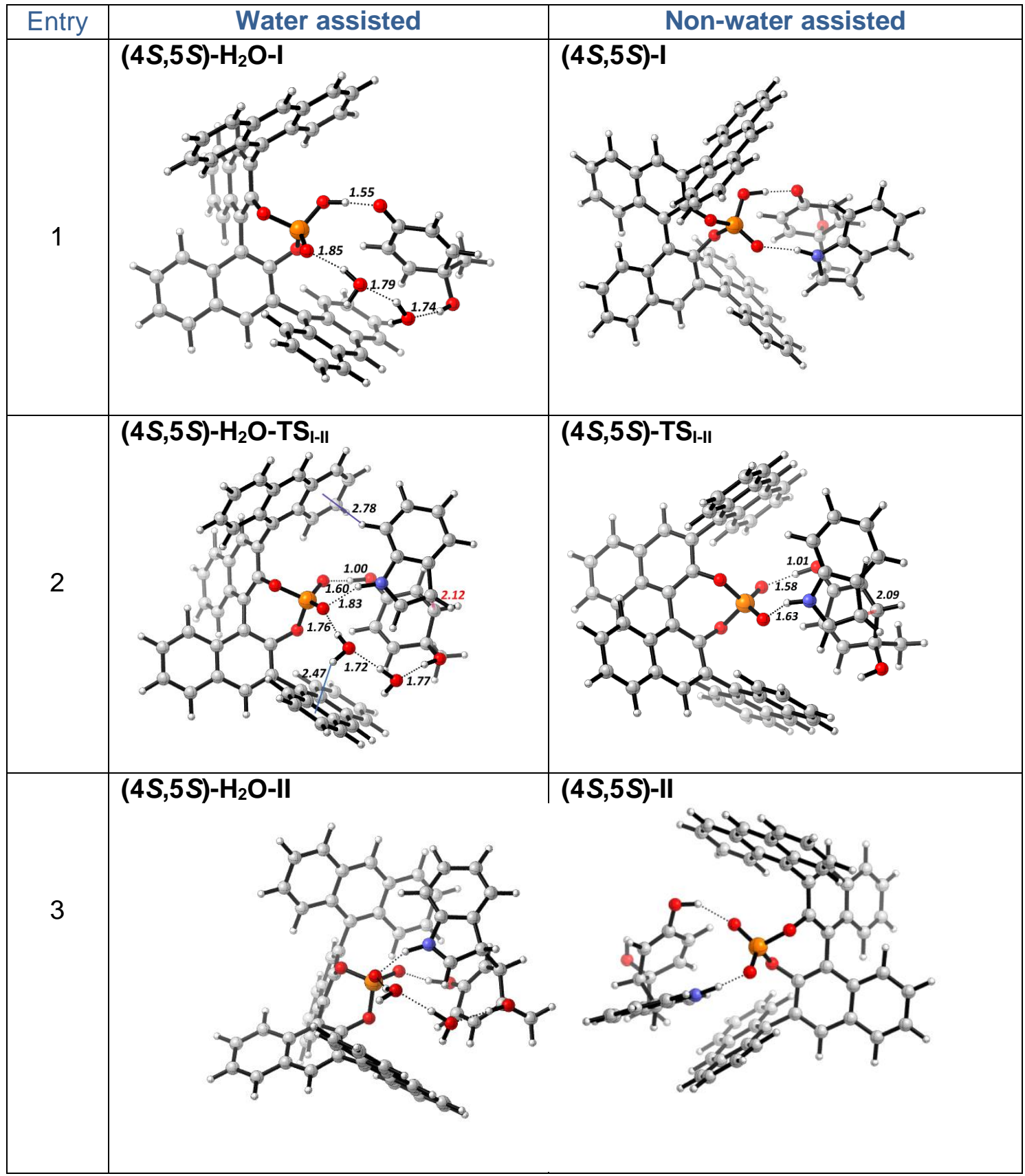

In Table 2 are shown all the calculated energies $(\Delta \mathrm{G}, \mathrm{kcal} / \mathrm{mol}$, gas phase) for the water assisted and the non-water assisted structures. A comparison of the energies of activation (in gas phase) and the structures of transition states of the Friedel-Crafts reaction indicates that the network of water molecules favors (by $9 \mathrm{kcal} / \mathrm{mol}$ ) the $(1,4)$-addition of indole $\mathbf{2 a}$ to $\mathbf{1 a}$ (entry 2 and 5 , Table 2). This effect might be due to the modulation of the acidity of phosphoric acid $\mathbf{1 2 h}$, which translates into an increase of the nucleophilicity of indole at its $\mathrm{C}_{3}$ position. 
Table 2. Calculated energies ( $\Delta \mathrm{G}, \mathrm{kcal} / \mathrm{mol}$, gas phase) for the non-assisted and for the water-assisted phosphoric acid-promoted $[(R)-\mathbf{1 2 h}] \mathrm{FC}$ reaction between $p$-quinol 1a (Re-face-pro- $S \mathrm{C} \beta)$ and indole 2a. ${ }^{\mathrm{a}}$

\begin{tabular}{|c|c|c|c|c|}
\hline & & & Structures & $\Delta \mathbf{G}(\mathrm{kcal} / \mathrm{mol})$ \\
\hline 1 & \multirow{3}{*}{$\mathrm{H}_{2} \mathrm{O}$-assisted } & \multirow{3}{*}{ Re face-pro-S $C \beta$} & $(4 S, 5 S)-\mathrm{H}_{2} \mathrm{O}-\mathbf{I}$ & 0.0 \\
\hline 2 & & & $(4 S, 5 S)-\mathbf{H}_{2} \mathbf{O}-\mathbf{T S}_{\mathbf{I - I I}}$ & 41.1 \\
\hline 3 & & & $(4 S, 5 S)-\mathrm{H}_{2} \mathrm{O}-\mathrm{II}$ & 5.2 \\
\hline 4 & \multirow{3}{*}{ Non-water assisted } & \multirow{3}{*}{ Re face- pro-S $C \beta$} & $(4 S, 5 S)-\mathbf{I}$ & 0.0 \\
\hline 5 & & & $(4 S, 5 S)-\mathbf{T S}_{\text {I-II }}$ & 50.5 \\
\hline 6 & & & $(4 S, 5 S)-$ II & 16.6 \\
\hline
\end{tabular}

${ }^{a}$ Energies were computed at the $\mathrm{wB} 97 \mathrm{XD} / 6-31 \mathrm{G}^{*}$ level in gas phase. ${ }^{\mathrm{b}}$ The activation energies are shown in italic blue.

As shown in Figure 2, the approach to the rear face of $p$-quinol 1a is blocked by steric interference of the methyl group. In contrast, the coordination of water molecules opens a cavity in the front face that can be occupied by indole 2a. Without disturbing the water-mediated $\mathrm{H}$-bonding network, indole $\mathrm{N}-\mathrm{H}$ group also binds the phosphoric acid basic site.

Figure 2. Representation of the $(R)$-12h phosphoric acid-complex with $p$-quinol $\mathbf{1 a}$ and $2 \cdot \mathrm{H}_{2} \mathrm{O}$ (wB97XD/PCM $\left.\left(\mathrm{CH}_{3} \mathrm{CN}\right) / 6-31 \mathrm{G}^{*} / / \mathrm{wB} 97 \mathrm{XD} / 6-31 \mathrm{G}^{*}\right)$. Relevant atom distances (in $\AA$ ) are shown.

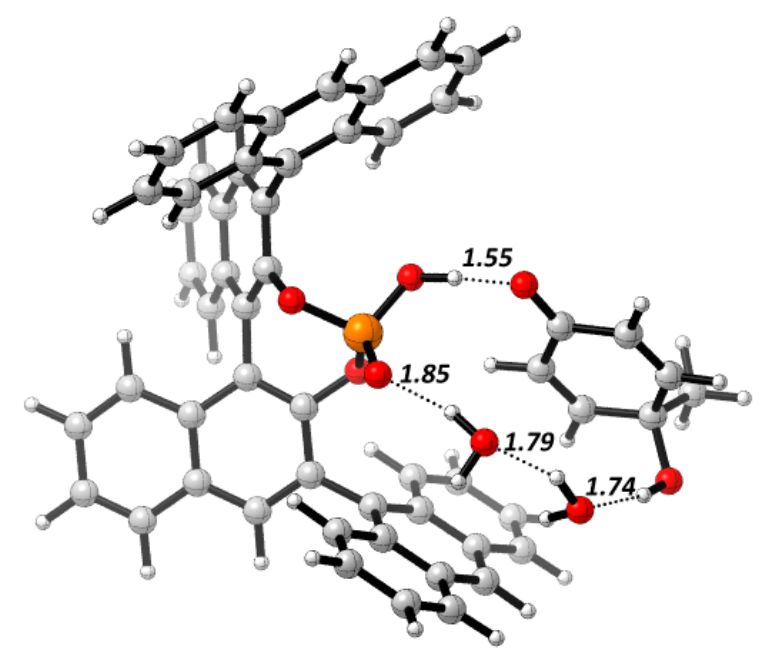

\section{COMPUTATIONAL STUDIES: DESYMMETRIZATION OF THE $p$-QUINOL}

In Table 3 are shown the water assisted diastereotopic approaches computed: entry 1) p-quinol associated with $(R)-\mathbf{1 2} \mathbf{h}(\mathbf{I})$, entry 2 ) the transitions state that incorporated the indole (TS final adduct 3a associated (R)-12h (II). The named (4S,5S) correspond to the Re face-pro-S $C \beta$ approach and $(4 R, 5 R)$ to the Si face-pro- $R C \beta$ approach. 
Table 3. Representation of the structures corresponding to the water-assisted, phosphoric acid-promoted $(R)$-12h Friedel-Crafts-type reaction between $p$-quinol $\mathbf{1 a}$ and indole $\mathbf{2 a}$ computed at the wB97XD/PCM $\left(\mathrm{CH}_{3} \mathrm{CN}\right) / 6-31 \mathrm{G}^{*} / \mathrm{wB} 97 \mathrm{XD} / 6-31 \mathrm{G}^{*}$ level. Relevant atom distances (in $\AA$ ) are shown.

Entry Water assisted complex

In Table 4 are shown all the calculated energies (in $\mathrm{CH}_{3} \mathrm{CN}$ ) for the water assisted $\mathrm{FC}$ reaction promoted by $(R)$-12h between $p$-quinol $1 \mathrm{a}$ and indole 2a for both diastereomeric approaches $(4 R, 5 R) \cdot(R) \mathbf{1 2 h}$ and $(4 S, 5 S) \cdot(R) \mathbf{1 2 h}$. As shown the Si face-pro- $R \mathrm{C} \beta$ approach of indole $\left[(4 R, 5 R)-\mathrm{H}_{2} \mathrm{O}-\right.$ TS the corresponding Re face-pro-S $C \beta$ counterpart (Table 3, entry 2) $\left[(4 S, 5 S)-\mathrm{H}_{2} \mathrm{O}-\mathrm{TS}_{\mathrm{I}-11}\right]$. Although the reactions are exergonic, transition state leading to the $(4 S, 5 S)$ enantiomer $(\Delta G-15.3 \mathrm{kcal} / \mathrm{mol},(4 S, 5 S)$ $\mathrm{H}_{2} \mathrm{O}$-II, table 3 , entry 3$)$ is $3.5 \mathrm{kcal} / \mathrm{mol}$ more stable than the one giving the $(4 R, 5 R)$ enantiomer $(\Delta \mathrm{G}$ $11.8 \mathrm{kcal} / \mathrm{mol},(4 R, 5 R)-\mathrm{H}_{2} \mathrm{O}-\mathrm{II}$, Table 3 , entry 6$)$. 
Table 4. Calculated energies (in $\mathrm{CH}_{3} \mathrm{CN}$ ) for the water assisted $\mathrm{FC}$ reaction promoted by $(R)-\mathbf{1 2} \mathbf{h}$ between $p$-quinol $\mathbf{1} \mathbf{a}$ and indole $\mathbf{2 a}$.

\begin{tabular}{|c|c|c|c|}
\hline Entry & & Structures $^{a}$ & $\Delta \mathbf{G}(\mathrm{kcal} / \mathrm{mol})^{\mathbf{b}}$ \\
\hline 1 & \multirow{3}{*}{ Re face-pro-S $C \beta^{c}$} & $(4 S, 5 S)-\mathrm{H}_{2} \mathrm{O}-\mathrm{I}$ & 2.4 \\
\hline 2 & & $(4 S, 5 S)-\mathbf{H}_{2} \mathbf{O}-\mathbf{T S}_{\mathbf{I - I I}}$ & $5.3(2.9)^{d}$ \\
\hline 3 & & $(4 S, 5 S)-\mathrm{H}_{2} \mathrm{O}-\mathrm{II}$ & -15.3 \\
\hline 4 & \multirow{3}{*}{ Si face-pro-S $C \beta^{c}$} & $(4 R, 5 R)-\mathrm{H}_{2} \mathrm{O}-\mathrm{I}$ & 0.0 \\
\hline 5 & & $(4 R, 5 R)-\mathbf{T S}_{\text {I-II }}$ & $9.2(9.2)^{d}$ \\
\hline 6 & & $(4 R, 5 R)-\mathrm{H}_{2} \mathrm{O}-\mathrm{II}$ & -11.8 \\
\hline
\end{tabular}

a $(\overline{4 S, 5 S)}$ and $(4 \mathrm{R}, 5 \mathrm{R})$ descriptors refer to the configuration of the final product $\mathbf{3 a}$ obtained. $\mathrm{b}$ Energies (in $\mathrm{kcal} / \mathrm{mol}$ ) were computed at the wB97XD/PCM $\left(\mathrm{CH}_{3} \mathrm{CN}\right) / 6-31 \mathrm{G}^{*} / / \mathrm{wB} 97 \mathrm{XD} / 6-31 \mathrm{G}^{*}$ level and refers to $(4 R, 5 R)-\mathrm{H}_{2} \mathrm{O}-\mathrm{I}$. ${ }^{\circ}$ Both approaches are obtained from $(R)$-phosphoric acid and are diastereomeric. ${ }^{d}$ Activation energies are shown in brackets.

Interestingly, it was found that the presence of water molecules, not only forms a more compact cavity in the transition state $(4 S, 5 S)-\mathrm{H}_{2} \mathrm{O}-\mathrm{TS}_{\mathrm{I}-\mathrm{II}}(\mathrm{H}-\mathrm{-O}$ distances $1.76,1.72$ and $1.77 \AA)$ than in $(4 R, 5 R)$ $\mathrm{H}_{2} \mathrm{O}-\mathrm{TS}_{\mathrm{I}-\mathrm{II}}(\mathrm{H}-\mathrm{-O}$ distances $1.84,1.86$ and $1.99 \AA)$, but also these molecules interact with the aryl ring of the phosphoric acid catalyst through $\mathrm{OH}-\pi$ interactions $\left(2.47 \AA\right.$ for $(4 S, 5 S)-\mathrm{H}_{2} \mathrm{O}-\mathrm{TS}_{\mathrm{I}-\mathrm{II}}$ and $2.33 \AA$ for $\left.(4 R, 5 R)-\mathbf{H}_{2} \mathbf{O}-\mathbf{T S}_{--11}\right)$ (Figure 4, A and B). ${ }^{7}$ Moreover, a comprehensive analysis of both transition structures reveals that the lowest-energy transition state exhibits another important noncovalent interaction, namely edge-to-face $\pi-\pi$ interaction ( $2.78 \AA$ ) between the indole and an aryl ring of the catalyst (Figure $4 \mathrm{~A}$ ). These types of interactions have been recognized to play important roles in chemical and biological recognition processes ${ }^{8}$ and also in the enantioselectivity, as shown by the desymmetrization of $p$-quinol $\mathbf{1 a}$.

\footnotetext{
${ }^{7}$ Takahashi, O.; Kohno, Y.; Nishio, M. Chem. Rev. 2010, 110, 6049-6079.

${ }^{8}$ Review about non-covalent interactions: (a). Salonen, L. M.; Ellermann, M.; Diederich, F. Angew. Chem. Int. Ed. 2011, 50, 4808-4842. (b) Krenske, E. K.; Houk, K. N. Acc. Chem. Res. 2013, 46, 979-989. For examples in Stereoselective Organic reactions: (a) Calleja, J.; Gónzalez Pérez, A. B.; de Lera, A. R.; Álvarez, R.; Fañanas, F.; Rodríguez, F. Chem. Sci., 2014, 5, 996-1007. (b) Liu, C.; Besora, M.; Maseras, F. Chem Asian J. 2016, 2016, 11, 411-416.
} 
Figure 4. Representation of the transition structures corresponding to the water-assisted, phosphoric acidpromoted $(R)$-12h Friedel-Crafts-type reaction between $p$-quinol $\mathbf{1 a}$ and indole $\mathbf{2 a}$ : $(4 S, 5 S)-\mathbf{H}_{2} \mathbf{O}-\mathbf{T S}_{\mathbf{I}-\text { II }}$ and $(4 R, 5 R)-\mathrm{H}_{2} \mathrm{O}-\mathrm{TS}_{\mathrm{I}-\mathrm{II}}$. Relevant atom distances (in $\AA$ ) are shown.

A. $(4 S, 5 S)-\mathrm{H}_{2} \mathrm{O}-\mathrm{TS}_{\mathrm{I}-\mathrm{II}}$

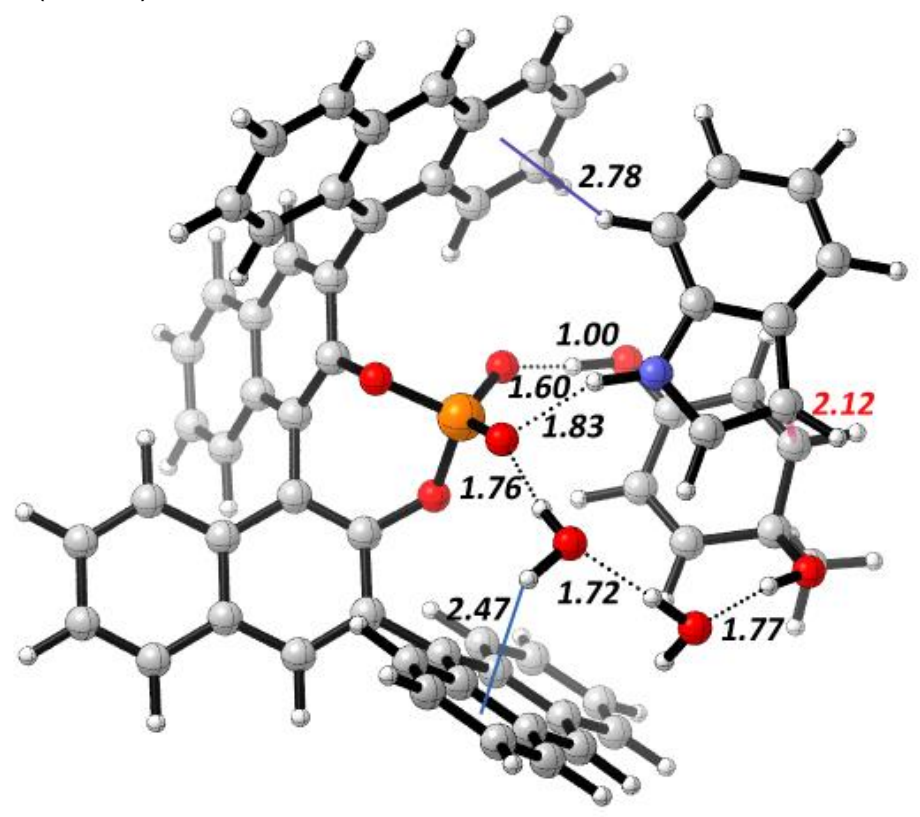

B. $(4 R, 5 R)-\mathrm{H}_{2} \mathrm{O}-\mathrm{TS}_{\mathrm{I}-\mathrm{II}}$

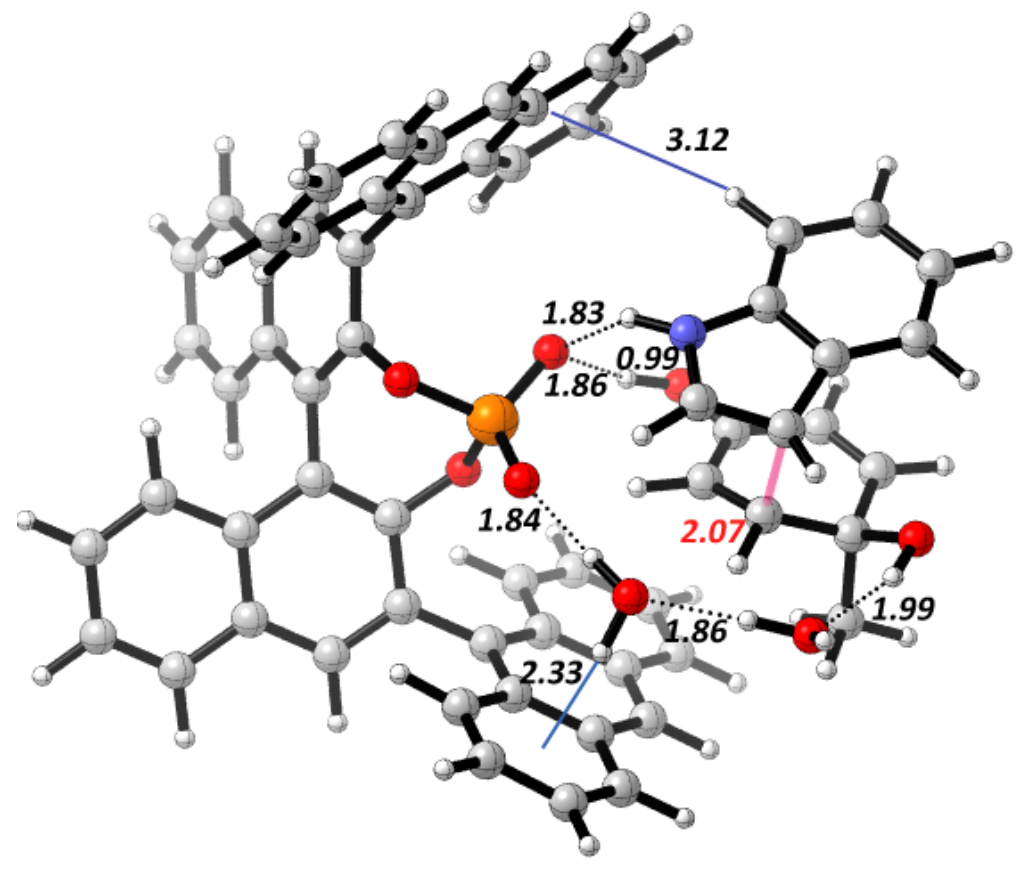


Table 3. Calculated energies ( $\mathrm{kcal} / \mathrm{mol}$, yellow in gas phase and blue in $\mathrm{CH}_{3} \mathrm{CN}$ ) for the water-assisted $(R)-\mathbf{1 2 h}$ catalized FC reaction of indole $\mathbf{2} \mathbf{a}$ to both enantiotopic double bonds of $p$-quinol $\mathbf{1 a}$.

\begin{tabular}{|c|c|c|c|c|c|c|c|c|}
\hline & EscF & $\mathrm{EsCF}_{\mathrm{SCV}}$ & H & s & G & & $\mathrm{G}\left(\mathrm{CH}_{3} \mathrm{CN}\right)$ & \\
\hline$(4 S, 5 S)-\mathrm{H}_{2} \mathrm{O}-\mathrm{I}$ & $-2150268,8$ & $-2149662,7$ & $-2149624,5$ & 98,3 & $-2149722,8$ & ref & $-2150308,6$ & 2,4 \\
\hline$(4 S, 5 S)-\mathrm{H}_{2} \mathrm{O}-\mathrm{TS}_{\mathrm{I}-\|}$ & $-2150232,5$ & $-2149623,9$ & $-2149586,7$ & 94,7 & $-2149681,4$ & 41,4 & $-2150300,5$ & 5,3 \\
\hline$(4 S, 5 S)-\mathrm{H}_{2} \mathrm{O}-\mathrm{II}$ & $-2150269,6$ & $-2149660,0$ & $-2149622,7$ & 94,9 & $-2149717,6$ & 5,2 & $-2150320,3$ & $-15,4$ \\
\hline$(4 R, 5 R)-\mathrm{H}_{2} \mathrm{O}-\mathrm{I}$ & $-2150269,4$ & $-2149662,7$ & $-2149624,5$ & 97,8 & $-2149722,3$ & 0,5 & $-2150309,7$ & ref \\
\hline$(4 R, 5 R)-\mathrm{H}_{2} \mathrm{O}-\mathrm{TS}_{\mathrm{I}-\|}$ & $-2150226,5$ & $-2149617,5$ & $-2149580,1$ & 94,9 & $-2149675,0$ & 47,8 & $-2150296,1$ & 9,2 \\
\hline$(4 R, 5 R)-\mathrm{H}_{2} \mathrm{O}-\mathrm{II}$ & $-2150265,6$ & $-2149656,3$ & $-2149618,7$ & 96,8 & $-2149715,5$ & 7,4 & $-2150318,6$ & $-11,8$ \\
\hline
\end{tabular}

Table 4. Calculated energies ( $\mathrm{kcal} / \mathrm{mol}$, in gas phase) for the FC reaction promoted by $(R)-\mathbf{1 2} \mathbf{h}$ phosphoric acid between $p$-quinol $\mathbf{1 a}$ and indole $\mathbf{2 a}$, water assisted or non-water assisted.

\begin{tabular}{|c|c|c|c|c|c|c|}
\hline & EscF & $E_{S C F}+Z V E$ & H & $s$ & G & \\
\hline$(4 S, 5 S)-\mathrm{H}_{2} \mathrm{O}-\mathrm{I}$ & $-2150268,8$ & $-2149662,7$ & $-2149624,5$ & 98,3 & $-2149722,8$ & ref \\
\hline$(4 S, 5 S)-\mathrm{H}_{2} \mathrm{O}-\mathrm{TS}_{\mathrm{I}-\|}$ & $-2150232,5$ & $-2149623,9$ & $-2149586,7$ & 94,7 & $-2149681,4$ & 41,4 \\
\hline$(4 S, 5 S)-\mathrm{H}_{2} \mathrm{O}-\mathrm{II}$ & $-2150269,6$ & $-2149660,0$ & $-2149622,7$ & 94,9 & $-2149717,6$ & 5,2 \\
\hline$(4 S, 5 S)-1$ & $-2054375,4$ & $-2053801,4$ & $-2053766,0$ & 94,8 & $-2053860,8$ & ref \\
\hline$(4 S, 5 S)-\mathrm{TS}_{\mathrm{I}-\|}$ & $-2054331,2$ & $-2053755,6$ & $-2053721,5$ & 88,8 & $-2053810,3$ & 50,5 \\
\hline$(4 S, 5 S)-I I$ & $-2054363,9$ & $-2053788,4$ & $-2053754,0$ & 90,3 & $-2053844,2$ & 16,6 \\
\hline
\end{tabular}


$(S, S)-\mathrm{H} 2 \mathrm{O}-\mathrm{I}$

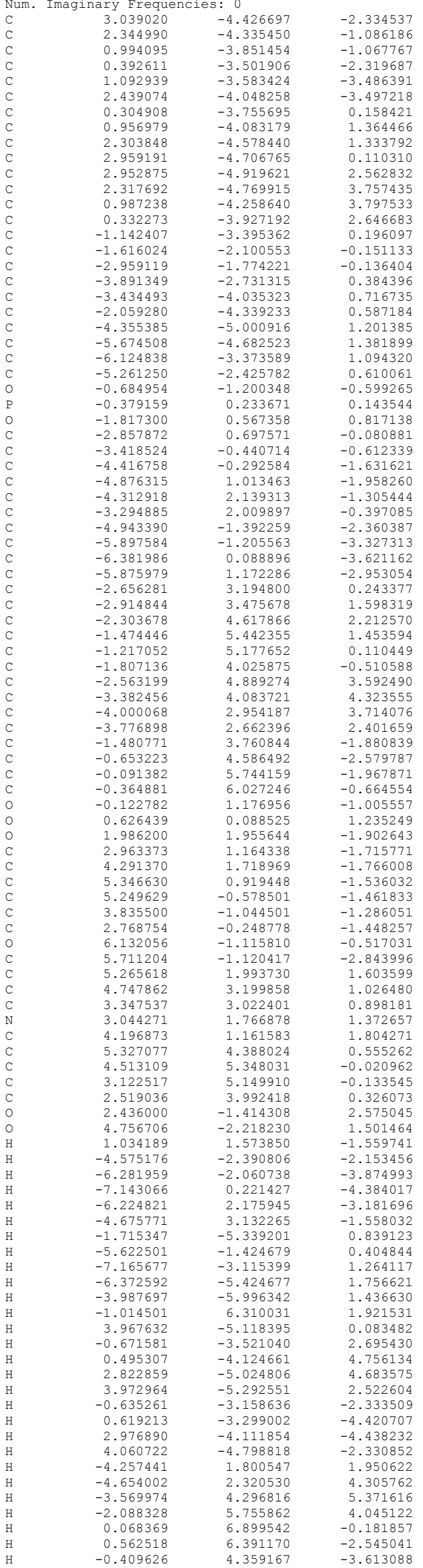

$\begin{array}{rrr}-1.880303 & 2.869247 & -2.348695 \\ 4.375713 & 2.796115 & -1.860917 \\ 6.350127 & 1.327709 & -1.458525 \\ 3.700743 & -2.103533 & -1.093898 \\ 1.761309 & -0.644518 & -1.403835 \\ 5.648070 & -1.289920 & 0.323228 \\ 1.737268 & -0.983985 & 2.039604 \\ 1.975915 & -2.042291 & 3.144717 \\ 3.877688 & -1.951306 & 1.864418 \\ 4.590035 & -3.065632 & 1.074746 \\ 5.620680 & -2.210174 & -2.840772 \\ 5.116876 & -0.705973 & -3.662642 \\ 6.764012 & -0.861303 & -2.985216 \\ 4.150147 & 0.183152 & 2.256550 \\ 6.294733 & 1.776733 & 1.852541 \\ 2.118181 & 1.346279 & 1.422919 \\ 6.398780 & 4.547977 & 0.640129 \\ 4.949269 & 6.271243 & -0.392167 \\ 1.450187 & 3.833741 & 0.217102 \\ 2.507942 & 5.915917 & -0.595491\end{array}$

$(S, S)-\mathrm{H}_{2} \mathrm{O}-\mathrm{TS}_{\mathrm{I}-\mathrm{II}}$

Num. Imaginary Frequencies: 1

1.775985 - 1.425327

$\begin{array}{ll}2.737673 & 3.425327 \\ 4.458206\end{array}$

$4.120269 \quad 3.714839$

$4.564840 \quad 5.000998$

$\begin{array}{ll}3.616916 & 5.987536 \\ 2.243248 & 5.699824 \\ 2.569854 & 2.107325\end{array}$

$\begin{array}{ll}2.243248 & 5.699824 \\ 2.569854 & 2.107325\end{array}$

$\begin{array}{ll}2.756927 & 1.523233\end{array}$

$\begin{array}{ll}4.783519 & 2.425609 \\ 4.979874 & 1.856860\end{array}$

$\begin{array}{ll}4.979874 & 1.856860\end{array}$

2.718221
2.760879
3.914131

3.914131

3.914131
5.222294

1.609588

6.112895

5.918221
-0.149858

-0.149858
-0.331024

-0.331024
0.619470

0.619470
-0.372992

$-1.191884$

$-2.566796$

-3.384387
-2.768376

-2.768376
-1.357656

-1.357656
-0.564326

$-4.796198$

-5.545121
-4.931977

-3.571558
-3.179895

-3.179895
-2.828808

-2.828808
-3.437184

-3.437184
-4.390587

-4.732803
-4.114475

$-5.673390$

$-5.973291$

$-5.331188$

-4.427435
-3.078769

$-3.533426$

-3.259229
-2.560654

$-2.560654$

-2.101346
-2.346203

-2.346203
-4.279275

-4.279275
-4.704782

-4.704782
-4.424110

-4.424110
-3.725807

$-1.388625$

$-0.912125$

-1.111870
-1.809938

-1.809938
-1.842791

0.918182

1.520860

2. 923679

3.679766
3.106049

3.106049
1.700437

3. 526321

2.788406

1.396467

0.786206
1.144326

1.932109

3.330132

3.895272

2.539376

0.826551

$-3.936862$

$-5.552980$

$-6.693242$

$-6.693242$

-6.146111
-4.879304

-4.879304
-0.889295

1.356761
1.568749

0.164108

$-0.390426$

0.354809
2.083970

-.083970
-0.029348

0.012493

1.198629

0.160073

0.108394

$-1.283678$

$-2.191551$

$-3.290403$

$-4.528979$

$-4.628105$

-3.547875
-3.188885

-3.188885
-4.266856

-4.266856
-5.510011

$-5.63329$

$-0.916921$

0.259346

1.506799

1.535267

0.37676

$-0.865044$

0.440037
-0.672526

$-0.672526$

$-1.899164$

-1.994129
2.744204

2.907800

4.131920

5.148845

4.995848

3.764499

1.896831

2. 083800

3.298122

6.28948

5.043069

5.869497

4.631185
3.620933

3.620933
0.228251

0.228251
-3.693080

-3.693080
-3.923734

$-4.217662$

$-4.242380$

$-3.957952$

$-3.679682$

$-4.456864$

$-4.406909$

$-4.101052$

$-3.865097$

$-3.411901$

$-3.37942$

$-3.638379$

$-3.932250$

$-1.006717$

$-1.566085$

1.652273

$-2.940243$

$-2.774566$

$-0.613884$

1.394246
2.476809

2.476809
-5.575960

0.454462

0.723074

0.680348

0.373828

0.130606

0.163705

1.029923

1.169280

0.831154

$-1.205662$

$-1.877680$

$-1.977529$

$-1.577595$

-1.178292
-1.197143

$-2.469535$

$-0.182484$

$-0.182484$

$-0.832996$

0.225449

0.225449
1.383380

$-0.572928$

$-0.217581$

$-0.285632$

0.465591

0.429569

0.131103

0.276969

0.673108

0.952648

0.855564

$-0.745176$

$-0.417455$

$-1.402641$

$-2.144662$

$-1.831119$

$-3.205734$

$-3.946866$

$-3.662397$

$-2.635106$

0.332972

1.655638
2.351228

1.351228
1.701267

0.394318

0.394318
-0.302322

2.343637
3.625148

3. 625148

3. 694493

3.694493
-0.273456

$-0.273456$

-1.538042
-2.214468

$-2.214468$

-1.624016
0.840905

0.200047

1. 453482

1.524140

0.350817

$-0.889943$

-0.972031
2.800154

3.943994

3.882960

2.684531

$-2.264594$

-3.377166
-3.287942

$-2.083782$

2. 976868

1.714494
-2.020468

$-2.035140$

$-4.265546$

$-3.423267$

-1.639538
0.682290 


$\begin{array}{lrrr}\text { H } & -5.282351 & -2.241176 & 0.076470 \\ \mathrm{H} & -6.620538 & -4.162156 & 0.780124 \\ \mathrm{H} & -5.539557 & -6.356681 & 1.257339 \\ \mathrm{H} & -3.082177 & -6.575771 & 1.087245 \\ \mathrm{H} & -2.370472 & 6.083034 & 2.225643 \\ \mathrm{H} & 4.738432 & -4.492714 & 0.403996 \\ \mathrm{H} & -0.268049 & -3.614684 & 2.654892 \\ \mathrm{H} & 0.821337 & -4.045539 & 4.802281 \\ \mathrm{H} & 3.257332 & -4.583092 & 4.906879 \\ \mathrm{H} & 4.592501 & -4.661925 & 2.837225 \\ \mathrm{H} & 0.080443 & -3.224077 & -2.352533 \\ \mathrm{H} & 1.490787 & -3.159976 & -4.344268 \\ \mathrm{H} & 3.938902 & -3.608983 & -4.186501 \\ \mathrm{H} & 4.959147 & -4.140721 & -2.003542 \\ \mathrm{H} & -4.504254 & 0.967542 & 1.831331 \\ \mathrm{H} & -5.263460 & 1.300587 & 4.128950 \\ \mathrm{H} & -4.772773 & 3.428556 & 5.335314 \\ \mathrm{H} & -3.509737 & 5.222514 & 4.208697 \\ \mathrm{H} & -1.231464 & 6.979243 & 0.256844 \\ \mathrm{H} & -0.367109 & 6.668054 & -2.033160 \\ \mathrm{H} & -0.688465 & 4.489290 & -3.203680 \\ \mathrm{H} & -1.935784 & 2.674221 & -2.134767 \\ \mathrm{H} & 3.763693 & 3.407997 & -2.127870 \\ \mathrm{H} & 5.894354 & 2.443246 & -1.252968 \\ \mathrm{H} & 3.953890 & -1.444204 & -0.922604 \\ \mathrm{H} & 1.849186 & -0.421572 & -1.632738 \\ \mathrm{H} & 5.640080 & -0.490474 & 0.544937 \\ \mathrm{H} & 1.817718 & -0.686343 & 2.394686 \\ \mathrm{H} & 2.179197 & -1.791871 & 3.408369 \\ \mathrm{H} & 4.073117 & -1.349065 & 2.270201 \\ \mathrm{H} & 4.545116 & -2.304433 & 1.177724 \\ \mathrm{H} & 6.068756 & -1.069483 & -2.590073 \\ \mathrm{H} & 5.323455 & 0.339061 & -3.396483 \\ \mathrm{H} & 6.898496 & 0.498025 & -2.567367 \\ \mathrm{H} & 3.830101 & 0.484668 & 1.447979 \\ \mathrm{H} & 5.819049 & 2.256861 & 1.092139 \\ \mathrm{H} & 1.689476 & 1.592318 & 1.160020 \\ \mathrm{H} & 5.626619 & 5.225136 & 0.323496 \\ \mathrm{H} & 3.941848 & 6.998506 & -0.096563 \\ \mathrm{H} & 0.716700 & 4.189454 & 0.447979 \\ \mathrm{H} & 1.525389 & 6.484250 & -0.051461 \\ & & & \\ & & & \end{array}$

$(S, S)-\mathrm{H}_{2} \mathrm{O}-\mathrm{II}$

\begin{tabular}{|c|c|c|c|}
\hline 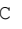 & -2.118132 & 5.240760 & 3.456987 \\
\hline C & -1.777331 & 4.877811 & 2.115851 \\
\hline 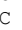 & -2.426510 & 3.750014 & 1.512108 \\
\hline c & -3.407738 & 3.047559 & 2.282954 \\
\hline C & -3.703033 & 3.422681 & 3.559320 \\
\hline C & -3.047381 & 4.535470 & 4.159917 \\
\hline C & -2.093163 & 3.379544 & 0.195625 \\
\hline C & -1.109057 & 4.088841 & -0.518882 \\
\hline 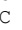 & -0.490895 & 5.236703 & 0.081370 \\
\hline C & -0.840906 & 5.604293 & 1.379710 \\
\hline 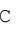 & 0.489818 & 5.965658 & -0.663168 \\
\hline C & 0.879355 & 5.552739 & -1.900336 \\
\hline C & 0.301593 & 4.387556 & -2.481268 \\
\hline C & -0.667419 & 3.693239 & -1.822773 \\
\hline C & -2.827614 & 2.265015 & -0.470009 \\
\hline . & -2.619591 & 0.909551 & -0.104096 \\
\hline C & -3.294709 & -0.140325 & -0.687528 \\
\hline C & -4.177005 & 0.133877 & -1.782909 \\
\hline C & -4.410252 & 1.487285 & -2.152258 \\
\hline $\mathrm{C}$ & -3.735257 & 2.526105 & -1.464568 \\
\hline c & -4.804347 & -0.888388 & -2.544854 \\
\hline c & -5.643976 & -0.578383 & -3.583559 \\
\hline C & -5.905780 & 0.768568 & -3.921970 \\
\hline $\mathrm{C}$ & -5.295569 & 1.776151 & -3.223421 \\
\hline c & -3.047619 & -1.521534 & -0.193588 \\
\hline 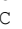 & -1.755877 & -2.006636 & -0.133834 \\
\hline $\mathrm{C}$ & -1.460190 & -3.347492 & 0.229975 \\
\hline C & -2.501722 & -4.175292 & 0.567378 \\
\hline c & -3.836725 & -3.707784 & 0.617815 \\
\hline C & -4.116529 & -2.360148 & 0.261666 \\
\hline C & -4.893994 & -4.553798 & 1.044557 \\
\hline C & -6.174150 & -4.078853 & 1.146658 \\
\hline C & -6.448836 & -2.727949 & 0.833393 \\
\hline C & -5.451531 & -1.892379 & 0.402181 \\
\hline 0 & -0.703142 & -1.216236 & -0.512942 \\
\hline P & -0.255699 & 0.102257 & 0.358639 \\
\hline 0 & -1.708291 & 0.635184 & 0.890539 \\
\hline C & -0.060376 & -3.858952 & 0.251794 \\
\hline C & 0.513068 & -4.241809 & 1.481829 \\
\hline C & 1.809815 & -4.854868 & 1.499548 \\
\hline $\mathrm{C}$ & 2.503534 & -5.029751 & 0.301616 \\
\hline C & 1.973944 & -4.595303 & -0.914008 \\
\hline C & 0.663765 & -4.010532 & -0.946656 \\
\hline C & 2.380012 & -5.252730 & 2.749212 \\
\hline C & 1.713437 & -5.049862 & 3.920483 \\
\hline C & 0.435409 & -4.416837 & 3.912675 \\
\hline C & -0.141099 & -4.024759 & 2.739103 \\
\hline C & 0.140292 & -3.610835 & -2.218795 \\
\hline C & 0.880020 & -3.736178 & -3.356464 \\
\hline C & 2.189755 & -4.295383 & -3.315445 \\
\hline C & 2.712450 & -4.724356 & -2.133646 \\
\hline 0 & 0.367243 & 1.057438 & -0.596058 \\
\hline 0 & 0.505192 & -0.295530 & 1.599778 \\
\hline 0 & 1.772539 & 1.480919 & -2.831403 \\
\hline C & 2.852564 & 0.923026 & -2.228414 \\
\hline C & 3.929274 & 1.644345 & -1.886980 \\
\hline C & 5.060460 & 1.016229 & -1.121190 \\
\hline C & 5.265657 & -0.490148 & -1.452576 \\
\hline C & 3.929472 & -1.183863 & -1.624760 \\
\hline
\end{tabular}

$\begin{array}{lrr}2.822439 & -0.529526 & -1.990669 \\ 4.852996 & 1.234586 & 0.440390 \\ 4.399634 & 2.631862 & 0.773018 \\ 3.083896 & 2.560191 & 1.232087 \\ 2.733194 & 1.192274 & 1.316703 \\ 3.701808 & 0.435101 & 0.927603 \\ 4.994554 & 3.873969 & 0.626576 \\ 4.249223 & 5.007263 & 0.958921 \\ 2.931269 & 4.902280 & 1.406990 \\ 2.313078 & 3.662054 & 1.547496 \\ 6.050327 & -1.108985 & -0.447320 \\ 6.049269 & -0.607159 & -2.765057 \\ 2.388379 & -1.784065 & 2.904781 \\ 4.326572 & -2.639061 & 1.207521 \\ 1.028457 & 1.337282 & -2.204485 \\ -4.606698 & -1.927081 & -2.305295 \\ -6.109063 & -1.376416 & -4.154547 \\ -6.579065 & 0.999791 & -4.741735 \\ -5.471549 & 2.816520 & -3.483939 \\ -3.918902 & 3.557014 & -1.755864 \\ -2.295267 & -5.209099 & 0.832575 \\ -5.675924 & -0.856597 & 0.174325 \\ -7.459547 & -2.345991 & 0.941444 \\ -6.975704 & -4.731254 & 1.479217 \\ -4.664294 & -5.585312 & 1.298870 \\ -0.372055 & 6.477087 & 1.831136 \\ 3.483089 & -5.503762 & 0.319153 \\ -1.102351 & -3.524114 & 2.750352 \\ -0.077042 & -4.237069 & 4.852849 \\ 2.156417 & -5.354770 & 4.863564 \\ 3.364435 & -5.712139 & 2.743140 \\ -0.856996 & -3.189532 & -2.270372 \\ 0.469078 & -3.408640 & -4.306157 \\ 2.762315 & -4.384878 & -4.233339 \\ 3.704253 & -5.167513 & -2.091769 \\ -3.917587 & 2.200002 & 1.837802 \\ -4.444959 & 2.869978 & 4.127768 \\ -3.294988 & 4.817958 & 5.178827 \\ -1.617535 & 6.096725 & 3.902728 \\ 0.929010 & 6.850904 & -0.209674 \\ 1.639337 & 6.102989 & -2.447251 \\ 0.657099 & 4.033869 & -3.442950 \\ -1.089604 & 2.799416 & -2.266172 \\ 3.914565 & 2.719266 & -2.030803 \\ 6.001567 & 1.535351 & -1.337119 \\ 3.927861 & -2.266654 & -1.527066 \\ 1.884729 & -1.052482 & -2.158484 \\ 5.481324 & -1.629281 & 0.153874 \\ 1.624996 & -1.404262 & 2.420188 \\ 2.010100 & -2.489050 & 3.445163 \\ 3.717738 & -2.383836 & 1.941224 \\ 3.746637 & -3.145789 & 0.622591 \\ 6.150355 & -1.659984 & -3.042775 \\ 5.541462 & -0.068420 & -3.570717 \\ 7.052884 & -0.190169 & -2.628243 \\ 3.620568 & -0.643352 & 0.951666 \\ 5.776606 & 0.906740 & 0.926498 \\ 1.807539 & 0.790319 & 1.590880 \\ 6.011856 & 3.968397 & 0.258668 \\ 4.700829 & 5.989254 & 0.858412 \\ 1.280391 & 3.570318 & 1.866879 \\ 2.370947 & 5.800080 & 1.644557\end{array}$

$(R, R)-\mathrm{H}_{2} \mathrm{O}-\mathrm{I}$

\begin{tabular}{|c|c|c|c|}
\hline $\mathrm{C}$ & -2.492964 & 4.887652 & 3.693036 \\
\hline C & -2.212267 & 4.595269 & 2.321246 \\
\hline $\mathrm{C}$ & -2.904341 & 3.514289 & 1.682695 \\
\hline C & -3.858085 & 2.776602 & 2.454774 \\
\hline C & -4.096673 & 3.084606 & 3.760526 \\
\hline C & -3.403832 & 4.156491 & 4.393286 \\
\hline C & -2.631838 & 3.214177 & 0.335582 \\
\hline$C$ & -1.690628 & 3.968515 & -0.389604 \\
\hline & -1.008912 & 5.053046 & 0.257843 \\
\hline & -1.282359 & 5.337111 & 1.594475 \\
\hline & -0.056798 & 5.820658 & -0.485319 \\
\hline$C$ & 0.206478 & 5.536336 & -1.790527 \\
\hline & -0.450327 & 4.444685 & -2.430364 \\
\hline & -1.358942 & 3.686704 & -1.755025 \\
\hline & -3.331150 & 2.074536 & -0.323581 \\
\hline & -2.965721 & 0.740266 & -0.007619 \\
\hline & -3.547918 & -0.364688 & -0.590059 \\
\hline & -4.519831 & -0.156086 & -1.622664 \\
\hline & -4.920868 & 1.173771 & -1.932420 \\
\hline & -4.317879 & 2.264188 & -1.255913 \\
\hline & -5.077340 & -1.220013 & -2.381847 \\
\hline & -6.008555 & -0.977475 & -3.358571 \\
\hline & -6.434914 & 0.340956 & -3.636223 \\
\hline & -5.896059 & 1.390568 & -2.940727 \\
\hline & -3.096192 & -1.722434 & -0.177458 \\
\hline & -1.749333 & -2.019071 & -0.218833 \\
\hline & -1.225729 & -3.297737 & 0.101645 \\
\hline & -2.108279 & -4.276560 & 0.483285 \\
\hline & -3.494706 & -4.016875 & 0.614812 \\
\hline & -3.999030 & -2.722085 & 0.308296 \\
\hline & -5.379153 & -2.467696 & 0.531342 \\
\hline & -6.212047 & -3.454338 & 0.991650 \\
\hline & -5.715813 & -4.751223 & 1.257243 \\
\hline & -4.385578 & -5.020975 & 1.076872 \\
\hline & -0.860554 & -1.055219 & -0.625795 \\
\hline & -0.508048 & 0.153968 & 0.429704 \\
\hline & -1.993007 & 0.558549 & 0.951182 \\
\hline
\end{tabular}




\begin{tabular}{|c|c|c|}
\hline 0.958423 & -3.807745 & 1.220510 \\
\hline 2.346219 & -4.169047 & 1.148701 \\
\hline 2.970072 & -4.254417 & -0.094434 \\
\hline 2.278756 & -3.983472 & -1.273911 \\
\hline 0.889655 & -3.631376 & -1.214680 \\
\hline 3.060943 & -4.443322 & 2.357756 \\
\hline 2.442663 & -4.370343 & 3.569706 \\
\hline 1.071931 & -3.981153 & 3.648689 \\
\hline 0.360843 & -3.698099 & 2.518649 \\
\hline 0.211366 & -3.367205 & -2.448121 \\
\hline 0.868053 & -3.423635 & -3.640718 \\
\hline 2.247288 & -3.775725 & -3.695387 \\
\hline 2.924392 & -4.058563 & -2.548111 \\
\hline 0.047602 & 1.234282 & -0.476835 \\
\hline 0.270612 & -0.336296 & 1.597038 \\
\hline 2.296100 & 2.630599 & 0.684862 \\
\hline 3.348442 & 3.078004 & -0.077578 \\
\hline 4.545146 & 2.710720 & 0.583800 \\
\hline 4.158392 & 2.013138 & 1.775060 \\
\hline 2.791937 & 1.980381 & 1.788111 \\
\hline 3.349458 & 3.754713 & -1.301748 \\
\hline 4.577964 & 4.066112 & -1.858910 \\
\hline 5.781539 & 3.709765 & -1.217931 \\
\hline 5.775075 & 3.036366 & -0.008482 \\
\hline 3.957072 & -0.946650 & -0.100553 \\
\hline 2.810001 & -0.478056 & -0.612779 \\
\hline 2.848047 & 0.407870 & -1.745565 \\
\hline 4.103683 & 0.717498 & -2.384550 \\
\hline 5.249286 & 0.292853 & -1.827757 \\
\hline 5.308264 & -0.682721 & -0.689803 \\
\hline 1.796801 & 0.968628 & -2.208826 \\
\hline 6.233218 & -0.209707 & 0.257407 \\
\hline 5.803233 & -2.033838 & -1.274860 \\
\hline 2.603248 & -1.081218 & 2.836403 \\
\hline 5.309326 & -1.109402 & 2.691276 \\
\hline 0.986833 & 0.956613 & -1.533084 \\
\hline-4.754074 & -2.236501 & -2.187316 \\
\hline-6.418906 & -1.806441 & -3.927354 \\
\hline-7.177932 & 0.518223 & -4.407766 \\
\hline-6.198825 & 2.411778 & -3.157101 \\
\hline-4.622332 & 3.276690 & -1.508325 \\
\hline-1.732519 & -5.268406 & 0.721633 \\
\hline-5.772077 & -1.475148 & 0.341462 \\
\hline-7.262524 & -3.237026 & 1.159541 \\
\hline-6.388939 & -5.524239 & 1.615048 \\
\hline-3.985986 & -6.008068 & 1.294542 \\
\hline-0.757917 & 6.156642 & 2.081219 \\
\hline 4.019014 & -4.539052 & -0.145504 \\
\hline-0.668574 & -3.368492 & 2.595004 \\
\hline 0.597393 & -3.890904 & 4.620999 \\
\hline 2.992417 & -4.585669 & 4.480793 \\
\hline 4.112485 & -4.707698 & 2.286862 \\
\hline-0.842665 & -3.113660 & -2.424057 \\
\hline 0.336024 & -3.208080 & -4.562159 \\
\hline 2.748851 & -3.831853 & -4.656743 \\
\hline 3.969604 & -4.355720 & -2.579446 \\
\hline-4.394634 & 1.959147 & 1.984696 \\
\hline-4.820432 & 2.508258 & 4.328994 \\
\hline-3.606429 & 4.384468 & 5.435437 \\
\hline-1.959974 & 5.709458 & 4.164328 \\
\hline 0.451443 & 6.639169 & 0.017398 \\
\hline 0.927256 & 6.129846 & -2.345403 \\
\hline-0.214160 & 4.211584 & -3.464438 \\
\hline-1.831970 & 2.843118 & -2.243911 \\
\hline 4.081977 & 1.423055 & -3.205832 \\
\hline 6.208618 & 0.631374 & -2.207910 \\
\hline 3.914530 & -1.606671 & 0.759630 \\
\hline 1.867734 & -0.735100 & -0.149768 \\
\hline 5.948253 & -0.507682 & 1.151874 \\
\hline 1.847966 & -0.720517 & 2.326776 \\
\hline 2.264635 & -1.905035 & 3.208621 \\
\hline 4.331336 & -0.995843 & 2.754838 \\
\hline 5.668306 & -0.656111 & 3.461567 \\
\hline 5.866524 & -2.765232 & -0.463528 \\
\hline 5.130179 & -2.408661 & -2.051679 \\
\hline 6.804303 & -1.891458 & -1.690924 \\
\hline 2.113239 & 1.528371 & 2.496309 \\
\hline 4.814860 & 1.575380 & 2.511549 \\
\hline 1.332259 & 2.559944 & 0.374295 \\
\hline 6.704683 & 2.743502 & 0.469109 \\
\hline 6.728158 & 3.968378 & -1.684474 \\
\hline 2.416525 & 4.011749 & -1.792511 \\
\hline 4.615213 & 4.594052 & -2.808009 \\
\hline
\end{tabular}

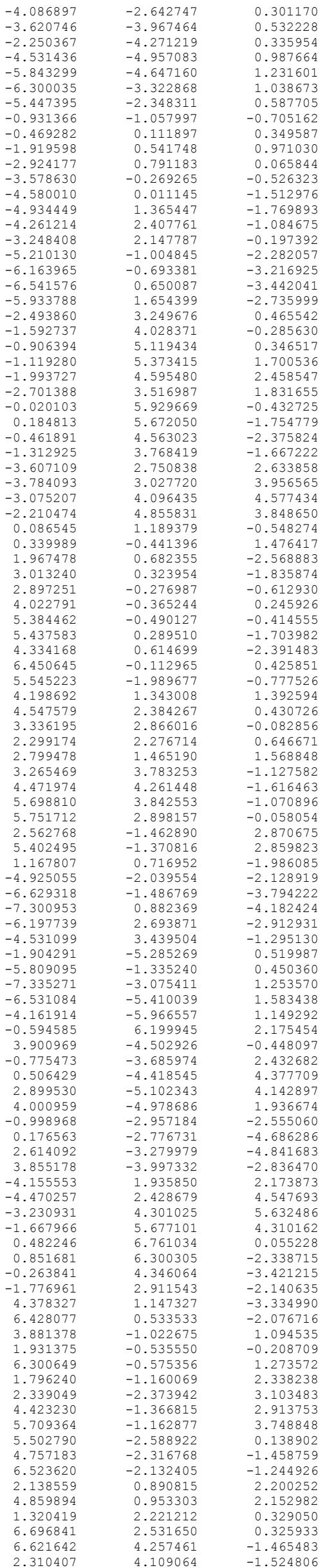


4.468150

4.985931

$-2.425291$

$(R, R)-\mathrm{H}_{2} \mathrm{O}$-II

Num. Imaginary Frequencies: 0

$\begin{array}{lll}\text { C } & 5.657414 & \\ \text { C } & 4.426267 & \\ \text { C } & 3.269709 & \\ \text { C } & 3.271335 & \\ \text { C } & 4.518074 & \\ \text { C } & 5.689664 & \\ \text { N } & 2.159498 & \\ \text { C } & 2.544374 & \\ \text { C } & 4.017007 & \\ \text { C } & 4.352971 & -0.5 \\ \text { C } & 3.894690 & -0.0 \\ \text { C } & 4.766638 & -0.8 \\ \text { C } & 6.193304 & -0.5 \\ \text { C } & 6.694323 & -0.2 \\ \text { C } & 5.851390 & -0.3 \\ \text { O } & 4.460372 & -1.2 \\ \text { O } & 6.394673 & \\ \text { C } & 5.952459 & -1.7 \\ \text { D } & -0.209408 & \end{array}$

-0.624363
0.274223

$-1.057540$

$-1.927077$

-1.392642
-2.257469

$-3.639158$

-3.639158
-4.153232

$-3.270815$

-4.512744
-5.835092

$-6.339374$

$-5.523227$

0.076561
0.832664

2.247403

2.858715

2.120367

0.705688

0.249273

0.999644

2.400320

2.748739

2.022136

0.614528

-0.019310
-3.733153

$-3.107240$

$-3.481676$

$-4.509136$

$-5.152792$

$-6.169866$

-6.169866
-6.752487

$-6.330618$

$-5.358588$

$-2.076822$

$-2.762486$

$-1.832846$

$-1.138086$

$-2.318171$

$-3.013333$

0.073247

$-0.609216$

-1.523456
-3.949084

$-3.94908$

-4.171432
-3.479331

$-2.583423$

2.164848
4.414193

4.414193
3.525459

3.525459
-5.038129

$-6.776907$

$-7.526050$

-6.469776
-4.819552

-4.819552
-1.871311

$-1.871311$

-5.922166
-7.382670

$-6.494796$

$-4.105791$

$-0.870210$

3.931663
-0.806567

0.536199

2.981578

4.073374

$-1.091681$

2.511116

0.041116
3.821568

$-4.483989$

$-4.881132$

-4.881132
-3.670528

$-2.051051$
2.854122

2.794529

2.794529
3.596725

4.026832

3.667349

2.254102
1.599647

1.599647

1.525548
-0.016528

0.607609

$-0.812913$

.516216

$-0.245186$

$-1.299690$

0.635841

-1.721244
1.270875

0.186386

$-0.263092$

$-1.068804$

$-2.011771$

$-3.288995$

-4.267421
-4.007903

$-2.711968$

$-1.713568$

$-5.011187$

$-4.741024$

-3.443184
-2.456812

$-3.523195$

$-3.680463$

$-3.955320$

$-3.796717$

$-3.578930$

-3.604882
-3.795672

$-3.795672$

$-4.050831$

$-4.088331$

$-3.844719$

$-3.673929$

$-3.425774$

-0.349637
0.740587

0.740587
2.082556

2.294613

1. 218857

0.118664

1.457513

0.421837

$-0.904389$

$-1.167777$

0.533010

3.210696
3.978589

5.050239

5.319752

4. 567095

3.490551
5.816614

5.543503

4.474832

4.474832
3.719252

2.735069

2.735069

3.025078

4. 842036

$-0.75754$

0.655851

$-1.554180$

$-2.189563$

0.616406

2. 484459

3. 312793

-5.256116
-1.463634

$-1.463634$

$-5.224784$

$-5.998846$

6.134911

$-4.120296$

$-3.378640$

$-3.726024$

-4.199145
-4.265268

$-3.270302$

$-3.337131$

$-3.709779$

-4.010569
1.917976

1.917976
2.435039

2.435039
4.311059

4.311059
5.662993

5.662993
6.626855
$-0.911487$

$-0.465105$

$-2.268116$

$-2.716790$

$-2.044396$

-0.446753
0.598206

0.685524

0.576376

$-0.731999$

$-1.571734$

$-0.366302$

$-2.959002$

1.784751

1.784751
-0.750601
0.193564

0.190378

1.297147

$-0.778116$

0.302813

0.433166

0.611801

0.325423

$-0.199824$

1.338614

1.094646

0.604326

1.098293

1.006901

$-0.243489$

$-1.337007$

.405741

3. 527471

3.431574

$-2.699772$

$-3.841642$

2.562683

$-0.578194$

$-0.289403$

$-1.171187$

$-1.557663$

$-2.795018$

$-3.476345$

$-2.300576$

0.872918

0.371003

0.355289
0.299912

1.642153

2.370238

1.727112
-0.439929

$-1.749456$

$-2.401986$

$-1.730328$

2.504355

3.748736

3.748736

3.728456

2.982808

$-2.130374$

$-3.788225$

$-2.987386$

1.392043

0.668469

0.431129
1.301854

1.720221

1.308513

2.133147
-0.309977

2.495356

4.506249

.336766
2.122942

$-2.520767$

$-4.684280$

$-2.745483$

2.032180
4.389149

4.389149
5.499523

4.222487

0.068809

0.796231

-0.394521
-2.009098

6.807704

7.747324

3.769920
2.834857

2.834857
5.854724

1. 390485

2. 360734

.568512
4.447048

4.447048
5.417367

5.417367

7.507212

1.816969

4. 388044

1.149727

6.565220

6.644089

2.348001
4.578339

6.13748
4.25995

2.886983 


$\begin{array}{lrrr}\text { H } & 5.200171 & 0.334245 & -2.178978 \\ \mathrm{H} & 6.474639 & -0.669820 & -4.011706 \\ \mathrm{H} & \mathbf{6 . 0 7 0 8 0 6} & -3.032760 & -4.689683 \\ \mathrm{H} & 4.330205 & -4.359012 & -3.543207 \\ \mathrm{H} & -1.807386 & 5.839808 & 0.583817 \\ \mathrm{H} & -2.212645 & -5.544691 & 1.698932 \\ \mathrm{H} & 0.218027 & -2.816072 & -2.485465 \\ \mathrm{H} & -1.867696 & -3.188888 & -3.701996 \\ \mathrm{H} & -3.753935 & -4.439821 & -2.640048 \\ \mathrm{H} & -3.532082 & -5.282674 & -0.339199 \\ \mathrm{H} & 2.980677 & -3.573493 & 1.646208 \\ \mathrm{H} & 3.140044 & -4.430394 & 3.928181 \\ \mathrm{H} & 1.198775 & -5.576681 & 4.994960 \\ \mathrm{H} & -0.908138 & -5.857960 & 3.744156 \\ \mathrm{H} & 2.356519 & 3.270905 & -2.046522 \\ \mathrm{H} & 1.413445 & 4.221216 & -4.092012 \\ \mathrm{H} & -0.637304 & 5.638195 & -4.008127 \\ \mathrm{H} & -1.725236 & 6.106783 & -1.848233 \\ \mathrm{H} & -1.969146 & 5.534219 & 2.996099 \\ \mathrm{H} & -1.123656 & 4.525610 & 5.082186 \\ \mathrm{H} & 0.874075 & 3.033775 & 5.025784 \\ \mathrm{H} & 2.022423 & 2.566991 & 2.922017 \\ \mathrm{H} & -4.968405 & 1.912922 & 1.269168 \\ \mathrm{H} & -6.815826 & 0.343837 & 0.753955 \\ \mathrm{H} & -4.012593 & -2.989190 & 0.966454 \\ \mathrm{H} & -2.191144 & -1.434503 & 1.502823 \\ \mathrm{H} & -3.917691 & -1.875211 & -2.695853 \\ \mathrm{H} & -6.177923 & -0.448001 & -3.114935 \\ \mathrm{H} & -2.232890 & -0.374873 & -1.590033 \\ \mathrm{H} & -6.749266 & 2.448124 & -2.412773 \\ \mathrm{H} & -5.676177 & 4.450430 & -1.426266 \\ \mathrm{H} & -1.971043 & 2.322717 & -0.881624 \\ \mathrm{H} & -3.321059 & 4.382918 & -0.671603 \\ \mathrm{H} & -5.762972 & -1.547754 & -0.987352 \\ \mathrm{H} & -6.375165 & -3.237019 & 2.026763 \\ \mathrm{H} & -6.116336 & -1.803971 & 3.058237 \\ \mathrm{H} & -7.521150 & -1.885098 & 1.958527 \\ \mathrm{H} & & & \end{array}$

$(S, S)-\mathrm{TS}_{\mathrm{I}-\mathrm{II}}$

Num. Imaginary Frequencies: 1

\begin{tabular}{|c|c|c|c|}
\hline C & -0.532956 & 5.628169 & -1.553753 \\
\hline C & 0.034164 & 5.000636 & -0.399373 \\
\hline C & 1.112026 & 4.069573 & -0.569233 \\
\hline C & 1.563487 & 3.801876 & -1.902027 \\
\hline C & 0.984033 & 4.404749 & -2.979432 \\
\hline c & -0.079873 & 5.337248 & -2.805990 \\
\hline C & 1.678224 & 3.447323 & 0.559276 \\
\hline C & 1.170016 & 3.707760 & 1.845975 \\
\hline C & 0.091993 & 4.642584 & 2.004034 \\
\hline C & -0.445349 & 5.268711 & 0.881713 \\
\hline c & -0.421042 & 4.901546 & 3.314445 \\
\hline C & 0.089813 & 4.265107 & 4.404041 \\
\hline C & 1.143251 & 3.318121 & 4.248811 \\
\hline C & 1.662996 & 3.048183 & 3.018844 \\
\hline C & 2.795513 & 2.471745 & 0.397856 \\
\hline C & 2.519845 & 1.169524 & -0.094981 \\
\hline C & 3.482500 & 0.188267 & -0.226714 \\
\hline C & 4.810084 & 0.476916 & 0.228143 \\
\hline C & 5.112338 & 1.789320 & 0.690909 \\
\hline C & 4.086463 & 2.766933 & 0.752338 \\
\hline C & 5.837484 & -0.504157 & 0.273428 \\
\hline C & 7.098591 & -0.187285 & 0.709117 \\
\hline C & 7.406790 & 1.127157 & 1.128126 \\
\hline C & 6.431074 & 2.088793 & 1.123276 \\
\hline C & 3.083607 & -1.142054 & -0.768742 \\
\hline C & 2.014832 & -1.796083 & -0.191855 \\
\hline c & 1.544911 & -3.063652 & -0.631282 \\
\hline C & 2.201808 & -3.667169 & -1.672911 \\
\hline C & 3.279232 & -3.028350 & -2.338975 \\
\hline C & 3.712887 & -1.742688 & -1.907395 \\
\hline C & 4.730898 & -1.091100 & -2.654803 \\
\hline C & 5.313116 & -1.701578 & -3.736123 \\
\hline C & 4.909813 & -2.995793 & -4.136697 \\
\hline C & 3.910308 & -3.638680 & -3.454743 \\
\hline 0 & 1.363139 & -1.207460 & 0.861712 \\
\hline P & 0.286044 & -0.043220 & 0.450479 \\
\hline 0 & 1.233570 & 0.915026 & -0.487248 \\
\hline C & 0.364691 & -3.692867 & 0.031916 \\
\hline C & -0.862480 & -3.795059 & -0.651138 \\
\hline C & -1.986294 & -4.408108 & -0.001350 \\
\hline C & -1.857633 & -4.874704 & 1.305002 \\
\hline C & -0.649703 & -4.772999 & 1.995745 \\
\hline $\mathrm{C}$ & 0.484650 & -4.180550 & 1.348994 \\
\hline C & -3.224861 & -4.532586 & -0.713134 \\
\hline C & -3.345395 & -4.082627 & -1.995670 \\
\hline C & -2.238260 & -3.446406 & -2.635103 \\
\hline C & -1.051164 & -3.294838 & -1.982340 \\
\hline c & 1.714697 & -4.116733 & 2.078670 \\
\hline C & 1.804477 & -4.592534 & 3.352771 \\
\hline C & 0.672913 & -5.172639 & 3.993954 \\
\hline C & -0.515124 & -5.261184 & 3.333477 \\
\hline 0 & -0.744024 & -0.588421 & -0.488921 \\
\hline 0 & -0.097776 & 0.616677 & 1.740225 \\
\hline $\mathrm{N}$ & -2.869283 & 0.429787 & -1.587697 \\
\hline C & -3.485751 & 1.650322 & -1.853697 \\
\hline C & -4.863878 & 1.411981 & -1.995637 \\
\hline C & -5.072017 & 0.004148 & -1.668887 \\
\hline C & -3.772929 & -0.539324 & -1.566957 \\
\hline C & -2.914233 & 2.913181 & -1.967001 \\
\hline C & -3.779482 & 3.963243 & -2.239604 \\
\hline $\mathrm{C}$ & -5.160397 & 3.753610 & -2.385862 \\
\hline $\mathrm{C}$ & -5.715631 & 2.485571 & -2.257553 \\
\hline C & -5.407310 & 0.136325 & 0.385816 \\
\hline
\end{tabular}

\begin{tabular}{rrr}
-5.315720 & -1.282585 & \\
-3.912886 & -1.607197 & 1.945220 \\
-2.994100 & -0.668628 & 1.630843 \\
-3.312037 & 0.747680 & 1.435894 \\
-4.539465 & 1.117540 & 0.915655 \\
-5.823823 & -2.261680 & 0.051855 \\
-6.232824 & -1.335628 & 2.184045 \\
-2.451827 & 1.671091 & 1.786527 \\
-1.520006 & 1.291428 & 1.896160 \\
5.613270 & -1.519086 & -0.035760 \\
7.866207 & -0.954755 & 0.738167 \\
8.410871 & 1.365914 & 1.465543 \\
6.647766 & 3.098251 & 1.463448 \\
4.323354 & 3.758891 & 1.128667 \\
1.871749 & -4.643065 & -2.020599 \\
5.042763 & -0.093426 & -2.366920 \\
6.087714 & -1.183201 & -4.293462 \\
5.382882 & -3.469602 & -4.991480 \\
3.575816 & -4.625572 & -3.764569 \\
-1.264366 & 5.973935 & 1.006581 \\
-2.712418 & -5.340673 & 1.792018 \\
-0.230317 & -2.776837 & -2.463461 \\
-2.355097 & -3.060841 & -3.643420 \\
-4.280399 & -4.207871 & -2.535774 \\
-4.061054 & -5.018230 & -0.214387 \\
2.585695 & -3.679688 & 1.603179 \\
2.747245 & -4.529150 & 3.887433 \\
0.765523 & -5.546900 & 5.009107 \\
-1.384376 & -5.707197 & 3.809961 \\
2.376571 & 3.098326 & -2.047769 \\
1.337342 & 4.179143 & -3.981121 \\
-0.522920 & 5.813652 & -3.675599 \\
-1.337986 & 6.344348 & -1.406229 \\
-1.234318 & 5.615375 & 3.416883 \\
-0.311719 & 4.463669 & 5.393366 \\
1.524438 & 2.796879 & 5.121686 \\
2.443043 & 2.303493 & 2.912539 \\
-4.736768 & 2.171744 & 0.760203 \\
-6.428158 & 0.446877 & 0.174285 \\
-3.688466 & -2.657552 & 1.548606 \\
-2.005418 & -0.926793 & 1.990281 \\
-3.450075 & -1.555565 & -1.396131 \\
-5.945378 & -0.582297 & -1.919800 \\
-1.885748 & 0.241619 & -1.297448 \\
-6.786181 & 2.335771 & -2.364500 \\
-5.805275 & 4.599459 & -2.604444 \\
-1.847962 & 3.072144 & -1.841190 \\
-3.372829 & 4.964330 & -2.343323 \\
-5.084391 & -2.636228 & -0.447270 \\
-6.159797 & -2.324660 & 2.644118 \\
-5.948242 & -0.572583 & 2.912611 \\
-7.270728 & -1.173904 & 1.876113 \\
& & \\
\hline & &
\end{tabular}

$(S, S)-I I$

Num. Imaginary Frequencies: 0

\begin{tabular}{rrr}
-4.592516 & 0.971395 & 0.962928 \\
-5.388940 & -0.043672 & 0.200574 \\
-5.318358 & -1.463896 & 0.822473 \\
-3.928768 & -1.756260 & 1.339724 \\
-3.078204 & -0.794020 & 1.707945 \\
-3.454499 & 0.626382 & 1.598170 \\
-5.713582 & -2.445237 & -0.138696 \\
-6.318998 & -1.555681 & 1.977909 \\
-2.670523 & 1.547661 & 2.175924 \\
-4.951312 & -0.065696 & -1.357063 \\
-4.775452 & 1.342130 & -1.857657 \\
-3.404081 & 1.599120 & -1.919294 \\
-2.726771 & 0.392548 & -1.635315 \\
-3.561398 & -0.566764 & -1.409544 \\
-5.666643 & 2.371644 & -2.112042 \\
-5.148821 & 3.629630 & -2.429754 \\
-3.771487 & 3.859032 & -2.472276 \\
-2.861469 & 2.836580 & -2.212315 \\
-0.208801 & 0.579268 & 1.679755 \\
0.268770 & -0.037298 & 0.412135 \\
-0.671832 & -0.613647 & -0.610314 \\
1.373324 & -1.172473 & 0.836158 \\
2.080921 & -1.716340 & -0.204797 \\
3.144597 & -1.015233 & -0.733097 \\
3.828447 & -1.571839 & -1.863045 \\
3.448178 & -2.860264 & -2.334906 \\
2.374859 & -3.549289 & -1.713997 \\
1.669840 & -2.991268 & -0.680074 \\
4.849236 & -0.874980 & -2.564042 \\
5.480225 & -1.443207 & -3.640849 \\
5.127257 & -2.738388 & -4.082396 \\
4.129094 & -3.425677 & -3.443992 \\
3.477540 & 0.317077 & -0.151508 \\
2.477543 & 1.261419 & -0.035896 \\
2.687848 & 2.562475 & 0.491345 \\
3.950461 & 2.893060 & 0.908064 \\
5.010456 & 1.950735 & 0.870739 \\
4.774873 & 0.641505 & 0.362431 \\
6.296549 & 2.281352 & 1.371996 \\
7.303512 & 1.353100 & 1.397630 \\
7.061635 & 0.042544 & 0.927637 \\
5.833461 & -0.304159 & 0.426316 \\
1.522671 & 3.486927 & 0.612280 \\
0.998639 & 4.114411 & -0.532965 \\
-0.147383 & 4.967889 & -0.412438 \\
-0.735306 & 5.152974 & 0.837479 \\
-0.235874 & 4.523950 & 1.975184 \\
0.914491 & 3.672454 & 1.867335 \\
& & \\
\hline
\end{tabular}




\begin{tabular}{|c|c|c|}
\hline 1.559213 & 3.924288 & -1.836992 \\
\hline 1.022626 & 4.531569 & -2.933815 \\
\hline-0.108260 & 5.390130 & -2.808387 \\
\hline-0.668622 & 5.602744 & -1.583993 \\
\hline-0.858080 & 4.697001 & 3.251355 \\
\hline-0.378682 & 4.060961 & 4.354563 \\
\hline 0.752473 & 3.201361 & 4.248749 \\
\hline 1.373822 & 3.011066 & 3.052107 \\
\hline 1.216281 & 0.969995 & -0.477971 \\
\hline 0.503326 & -3.678721 & -0.051373 \\
\hline-0.714522 & -3.798266 & -0.747080 \\
\hline-1.824675 & -4.460092 & -0.122768 \\
\hline-1.691020 & -4.962884 & 1.168984 \\
\hline-0.491266 & -4.847689 & 1.870679 \\
\hline 0.628681 & -4.202114 & 1.250906 \\
\hline-3.056644 & -4.591222 & -0.844180 \\
\hline-3.187589 & -4.094590 & -2.108284 \\
\hline-2.093036 & -3.414389 & -2.723161 \\
\hline-0.909083 & -3.264153 & -2.064095 \\
\hline 1.850435 & -4.121135 & 1.992258 \\
\hline 1.945768 & -4.632177 & 3.251755 \\
\hline 0.828364 & -5.265492 & 3.866280 \\
\hline-0.351358 & -5.370651 & 3.194326 \\
\hline-1.732254 & 1.237946 & 2.151698 \\
\hline 5.658542 & -1.316644 & 0.079713 \\
\hline 7.853299 & -0.699442 & 0.970881 \\
\hline 8.281066 & 1.615208 & 1.790809 \\
\hline 6.462237 & 3.286684 & 1.750769 \\
\hline 4.137535 & 3.883336 & 1.315466 \\
\hline 2.091304 & -4.529521 & -2.089349 \\
\hline 5.123923 & 0.123506 & -2.242544 \\
\hline 6.255913 & -0.890472 & -4.162517 \\
\hline 5.638392 & -3.178576 & -4.933165 \\
\hline 3.834445 & -4.415118 & -3.784557 \\
\hline-1.611336 & 5.792190 & 0.924638 \\
\hline-2.536615 & -5.462842 & 1.637581 \\
\hline-0.095997 & -2.717283 & -2.526199 \\
\hline-2.213549 & -3.002987 & -3.720977 \\
\hline-4.119724 & -4.220440 & -2.652873 \\
\hline-3.880262 & -5.118987 & -0.367278 \\
\hline 2.710778 & -3.643395 & 1.536946 \\
\hline 2.882258 & -4.555888 & 3.795632 \\
\hline 0.925070 & -5.666434 & 4.870731 \\
\hline-1.210490 & -5.854742 & 3.651401 \\
\hline 2.426458 & 3.280960 & -1.944452 \\
\hline 1.463710 & 4.369011 & -3.912663 \\
\hline-0.514080 & 5.875986 & -3.691117 \\
\hline-1.526315 & 6.262072 & -1.472088 \\
\hline-1.731521 & 5.340387 & 3.315366 \\
\hline-0.863619 & 4.192693 & 5.317111 \\
\hline 1.109992 & 2.679742 & 5.131289 \\
\hline 2.212820 & 2.328919 & 2.979558 \\
\hline-4.842409 & 2.021529 & 0.860884 \\
\hline-6.442425 & 0.254512 & 0.150769 \\
\hline-3.665942 & -2.804690 & 1.466642 \\
\hline-2.100764 & -1.032338 & 2.113666 \\
\hline-3.181834 & -1.552941 & -1.181169 \\
\hline-5.669598 & -0.698956 & -1.878735 \\
\hline-1.702081 & 0.209195 & -1.403261 \\
\hline-6.739346 & 2.211948 & -2.058304 \\
\hline-5.831152 & 4.446975 & -2.641545 \\
\hline-1.790029 & 3.009015 & -2.230200 \\
\hline-3.394073 & 4.848615 & -2.708292 \\
\hline-4.921858 & -2.895867 & -0.464557 \\
\hline-6.231839 & -2.528135 & 2.469994 \\
\hline-6.131976 & -0.764061 & 2.709156 \\
\hline 337933 & -1.453762 & $1.5891 \mathrm{~g}$ \\
\hline
\end{tabular}

